\title{
An Algebraic Family of Distributed Space-Time Codes for Wireless Relay Networks
}

\author{
Frédérique Oggier and Babak Hassibi \\ Department of Electrical Engineering \\ California Institute of Technology \\ Pasadena, CA 91125 \\ \{frederique, hassibi\}@systems.caltech.edu
}

\begin{abstract}
This paper studies the design of distributed spacetime codes for use in wireless relay networks. Earlier work suggested that a suitable family of codes can be obtained by using linear dispersion codes, provided the basis matrices were unitary. In this paper we construct an explicit algebraic family of such codes where full diversity is proved. The construction uses cyclotomic field theory and yields basis matrices that are indeed unitary. Simulation results show that the codes have better performance than codes designed earlier by ad hoc and random methods, and thus with less encoding complexity.
\end{abstract}

\section{INTRODUCTION}

There has been recent interest in looking for methods to exploit spatial diversity using the antennas of the different users in a wireless network [9], [10], [12], [6], [2], [8], [4], [7], [11], [1]. Many of these schemes use ideas from space-time coding, devised for point-to-point communications, and so are refered to as distributed space-time coding to acknowledge the fact that the various (relay) nodes in a wireless network must implement the code in a distributed fashion.

In [5] a particular framework for distributed space-time coding was proposed and the resulting error probability and diversity was analyzed. This system has the benefit that the relay nodes do not need to know their channel coefficients from the sender, and to the receiver, and that they are not required to decode their received signals. Instead, all they need to do is to perform simple linear operations on the signals they receive. It can therefore be easily shown that the relay nodes can readily implement a space-time linear dispersion code [3] in a distributed fashion. In fact, the probability of error (and hence diversity) behaves almost as that of a point-topoint multi-antenna system with the same number of transmit antennas as the network's relay nodes $\left(P_{e} \approx\left(\frac{\log p}{p}\right)^{n}\right.$, as opposed to $P_{e} \approx\left(\frac{1}{p}\right)^{n}$ ).

In order to guarantee that the different relay nodes and signals are treated in an equitable fashion and that the noise forwarded by the relay nodes to the receiver remains temporally white, in [5] it was argued that the basis matrices of the linear dispersion code must be unitary. However, no explicit code construction was proposed. The goal of this paper is

\footnotetext{
${ }^{1}$ This work was supported in part by the Swiss National Science Foundation grant PBEL2-110209 and by NSF grant CCR-0133818, by Caltech's Lee Center for Advanced Networking and by a grant from the David and Lucille Packard Foundation.
}

to give an algebraic family of such codes where full diversity can be proved. The construction relies heavily on the theory of cyclotomic fields. The novelty here, over earlier constructions of fully diverse linear dispersion codes, is that we obtain basis matrices that are unitary.

The rest of the paper is organized as follows. Section II introduces the network model and section III describes the distributed space-time coding framework. Section IV describes the code construction, section $\mathrm{V}$ establishes the full diversity and section VI gives a sample simulation. The paper concludes briefly with section VII.

\section{THE WIRELESS NETWORK MODEL}

Consider a wireless network with $R+2$ nodes which are placed randomly, independently according to some distribution. There are two distinguished nodes: the receiver and the transmitter. Other nodes are relays. Every node has a single antenna, it can transmit and receive, but not simultaneously. The channel from the transmitter to the $i$ th relay is denoted by $f_{i}$, while the one from the $i$ th relay to the receiver is denoted by $g_{i}$. Both channels are assumed independent complex Gaussian with zero-mean and unit-variance. The relays are not assumed to know the values of the fading coefficients $f_{i}$ and $g_{i}$. However, we assume that the receiver knows all the fading coefficients $f_{i}$ and $g_{i}$. In order for the receiver to estimate the $f_{i}$, a block of known symbols using the same space-time coding scheme is send. In other words, the relay nodes would act as if they were encoding data, but the receiver would solve for the equivalent channel matrix (rather than the transmitted symbols) since it knows the transmitted symbols.

Let $\left\{\mathbf{s}_{1}, \ldots, \mathbf{s}_{L}\right\}$ be the codebook of cardinality $L$. The transmission is done as follows: assume the transmitter wants to send the signal $\mathbf{s}=\left(s_{1}, \ldots, s_{T}\right)$, where $\mathbf{s}$ is normalized such that $\mathbf{s}^{*} \mathbf{s}=1$. From time 1 to $T$, the transmitter sends the signals $\sqrt{P_{1} T} s_{1}, \ldots, \sqrt{P_{1} T} s_{T}$ to each relay, where $P_{1}$ is the average power used for every transmission. The received signal at the $i$ th relay at time $\tau$ is given by $r_{i, \tau}=\sqrt{P_{1} T} f_{i} s_{\tau}+v_{i, \tau}$, with $v_{i, \tau}$ the complex Gaussian noise with zero mean and unit variance. From time $T+1$ to $2 T$, the $i$ th relay sends $t_{i, 1}, \ldots, t_{i, \tau}$ to the receiver. The received signal at the transmitter at time $T+\tau$ is given by $x_{\tau}=\sum_{i=1}^{R} g_{i} t_{i, \tau}+w_{\tau}$, where $\tau$ is the complex Gaussian noise with zero mean and unit variance (see Figure 1). Using bold face letters for designing 


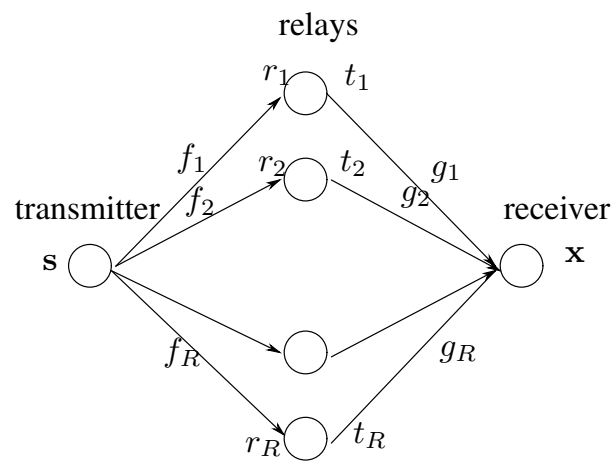

Fig. 1. The wireless network

vectors and assuming a coherence interval of $T$, we summarize the channel as follows:

$$
\mathbf{r}_{i}=\sqrt{P_{1} T} f_{i} \mathbf{s}+\mathbf{v}_{i}, \mathbf{x}=\sum_{i=1}^{R} g_{i} \mathbf{t}_{i}+\mathbf{w} .
$$

\section{Distributed SPACE-TIME CODING}

The idea behind Distributed Space-Time Coding is to design the transmit signal at every relay as a linear function of its received signal:

$$
\mathbf{t}_{i}=\sqrt{\frac{P_{2}}{P_{1}+1}} A_{i} \mathbf{r}_{i},
$$

where $A_{i}$ is an $T \times T$ matrix, $i=1, \ldots, R$, and $P_{2}$ is the average transmit power for one transmission at every relay node. See [5] for the detail on how to compute the normalization factor. In order to have an equitable protocol among different users and among different times instants, the matrices $A_{i}$ are assumed unitary. (As mentioned earlier, this also guarantees that the noise at the receiver, $\mathbf{w}$, remains temporally white.)

From (1) and (2), the received signal is given by

$$
\mathbf{x}=\sqrt{\frac{P_{1} P_{2} T}{P_{1}+1}} S H+W,
$$

with

$$
S=\left[A_{1} \mathbf{s} \cdots A_{R} \mathbf{s}\right], H=\left[\begin{array}{c}
f_{1} g_{1} \\
\vdots \\
f_{R} g_{R}
\end{array}\right]
$$

and

$$
W=\sqrt{\frac{P_{2}}{P_{1}+1}} \sum_{i=1}^{R} g_{i} A_{i} \mathbf{v}_{i}+\mathbf{w} .
$$

The $T \times R$ matrix $S$ works like the space-time code in a multiple-antenna system. It is called a distributed space-time code since it has been generated in a distributed way by the relay nodes.

The question that we address in this work is the design of the distributed space-time code, i.e., the design of the matrices $A_{i}$, for the case $T=R$. In [5], it has been shown that similarly to the multi antennas case, the full diversity criterion holds, that is, $S_{k}-S_{l}$ has to be full rank, for $S_{k} \neq S_{l}$ two distributed space-time codes. We show in next section how to build a family of unitary matrices $A_{i}$ such that the resulting space-time code is fully diverse. Note that considering the case $T=R$ is not a restriction, since if $R>T$, then the diversity is at most $T$, meaning we can use a $T \times T$ code. Similarly, if $R<T$, we can use a $R \times R$ code.

\section{A BASIS OF UNITARY MATRICES}

Let $\zeta=\zeta_{2^{n}}=e^{2 i \pi / 2^{n}}$ be a primitive $2^{n}$ th rooth of unity. We consider the cyclotomic field $\mathbb{Q}(\zeta)$ defined by

$$
\mathbb{Q}(\zeta)=\left\{x=\sum_{l=0}^{2^{n-1}-1} x_{l} \zeta^{l}, x_{l} \in \mathbb{Q}\right\} .
$$

For example, if $n=2, \mathbb{Q}(\zeta)=\mathbb{Q}(i)=\left\{x=x_{0}+\right.$ $\left.i x_{1}, x_{0}, x_{1} \in \mathbb{Q}\right\}$. The field $\mathbb{Q}(\zeta)$ has a structure of vector space of dimension $2^{n-1}$ over $\mathbb{Q}$. A $\mathbb{Q}$-basis is given by $\left\{1, \zeta, \ldots, \zeta^{2^{n-1}-1}\right\}$.

Lemma 1: For $n \geq 2$, the field $\mathbb{Q}(\zeta)$ is a vector space of dimension $2^{n-2}$ over $\mathbb{Q}(i)$.

Proof: We have that every $x$ in $\mathbb{Q}(\zeta)$ can be written as

$$
\begin{aligned}
x & =\sum_{l=0}^{2^{n-2}-1} x_{l} \zeta^{l}+\sum_{l=2^{n-2}}^{2^{n-1}-1} x_{l} \zeta^{l} \\
& =\sum_{l=0}^{2^{n-2}-1} x_{l} \zeta^{l}+\zeta^{2^{n-2}} \sum_{k=0}^{2^{n-2}-1} x_{k+2^{n-2}} \zeta^{k} \\
& =\sum_{l=0}^{2^{n-2}-1}\left(x_{l}+i x_{l+2^{n-2}}\right) \zeta^{l},
\end{aligned}
$$

since $\zeta^{2^{n-2}}$ is a fourth root of unity. Thus

$$
\left\{1, \zeta, \ldots, \zeta^{2^{n-2}-1}\right\}
$$

is a $\mathbb{Q}(i)$-basis of $\mathbb{Q}(\zeta)$.

Let $x \in \mathbb{Q}(\zeta)$, written in the $\mathbb{Q}(i)$-basis (4). We define the matrix $M_{x}$ of multiplication by $x$ in this $\mathbb{Q}(i)$-basis by

$$
\left(1, \zeta, \ldots, \zeta^{2^{n-2}-1}\right) M_{x}=\left(x, x \zeta, \ldots, x \zeta^{2^{n-2}-1}\right),
$$

where $M_{x}$ has coefficients in $\mathbb{Q}(i)$.

Let $m=2^{n-2}$. If $x=\sum_{l=0}^{m-1} x_{l} \zeta^{l}$, we have

$$
\begin{aligned}
x \zeta & =\sum_{l=0}^{m-1} x_{l} \zeta^{l+1} \\
& =\sum_{l=0}^{m-2} x_{l} \zeta^{l+1}+i x_{m-1} \\
& =i x_{m-1}+\sum_{k=1}^{m-1} x_{k-1} \zeta^{k}
\end{aligned}
$$


since $\zeta^{m}=\zeta^{2^{n}-2}$ is a fourth root of unity. Similarly,

$$
\begin{aligned}
x \zeta^{2} & =\sum_{l=0}^{m-1} x_{l} \zeta^{l+2} \\
& =\sum_{l=0}^{m-3} x_{l} \zeta^{l+2}+i x_{m-2}+i x_{m-1} \zeta \\
& =i x_{m-2}+i x_{m-1} \zeta+\sum_{k=2}^{m-1} x_{k-2} \zeta^{k} .
\end{aligned}
$$

More generally,

$$
\begin{aligned}
x \zeta^{t} & =\sum_{l=0}^{m-1} x_{l} \zeta^{l+t} \\
& =\sum_{l=0}^{m-t-1} x_{l} \zeta^{l+t}+i \sum_{l^{\prime}=1}^{t} x_{m-l^{\prime}} \zeta^{t-l^{\prime}} \\
& =i \sum_{l^{\prime}=1}^{t} x_{m-l^{\prime}} \zeta^{t-l^{\prime}}+\sum_{k=t}^{m-1} x_{k-t} \zeta^{k}
\end{aligned}
$$

We thus have that $M_{x}$ is given by

$$
\left(\begin{array}{ccccc}
x_{0} & i x_{m-1} & i x_{m-2} & \ldots & i x_{1} \\
x_{1} & x_{0} & i x_{m-1} & & i x_{2} \\
x_{2} & x_{1} & x_{0} & & i x_{3} \\
\vdots & \vdots & \vdots & & \vdots \\
x_{m-2} & x_{m-3} & x_{m-4} & & i x_{m-1} \\
x_{m-1} & x_{m-2} & x_{m-3} & \ldots & x_{0}
\end{array}\right) .
$$

Let

$$
G=\left(\begin{array}{ccccc}
0 & 0 & 0 & & i \\
1 & 0 & 0 & & \\
0 & 1 & & & \\
\vdots & & \ddots & \ddots & \\
& & & 1 & 0
\end{array}\right)
$$

Let $\mathbf{x}=\left(x_{0}, \ldots, x_{m-1}\right)$. The matrix $M_{x}$ can be written as

$$
M_{x}=\left[\begin{array}{llll}
G^{0} \mathbf{x} G \mathbf{x} \cdots G^{m-2} \mathbf{x} G^{m-1} \mathbf{x}
\end{array}\right] .
$$

We thus design the distributed space-time code described in (3) as

$$
A_{i}=G^{i-1}, i=1, \ldots, R, \quad S=\left[I \mathbf{s}, G \mathbf{s}, \ldots, G^{R-1} \mathbf{s}\right] .
$$

The matrices $A_{i}=G^{i-1}, i=1, \ldots, R$, are clearly unitary.

\section{THE DIVERSITY PROPERTY}

We now show that the space-time code given in the previous section satisfies the full diversity property.

Let $x, x^{\prime} \in \mathbb{Q}(\zeta)$. Note first that $M_{x}-M_{x^{\prime}}=M_{x-x^{\prime}}$. Thus

$$
\operatorname{det}\left(M_{x}-M_{x^{\prime}}\right)=\operatorname{det}\left(M_{y}\right), x \neq x^{\prime}, y \in \mathbb{Q}(\zeta) .
$$

In order to show that $\operatorname{det}\left(M_{x}\right)$ is different from 0 for any $x \neq 0$, we compute the eigenvalues of $M_{x}$. By definition of $M_{x}(5), x$ itself is an eigenvalue associated to the eigenvector $\left(1, \zeta, \ldots, \zeta^{2^{n-2}-1}\right)$. We now compute the other eigenvalues.
Recall that $m=2^{n-2}$, and let $r$ be an element of order $m$ modulo $2^{n}$, that is $m$ is the smallest positive integer such that

$$
r^{m} \equiv 1\left(\bmod 2^{n}\right)
$$

Define $\sigma$ to be the following map

$$
\sigma: \zeta \mapsto \zeta^{r},
$$

which is extended by linearity to yield

$$
\sigma\left(\sum_{l=0}^{m-1} x_{i} \zeta^{i}\right)=\sum_{l=0}^{m-1} x_{i} \sigma\left(\zeta^{i}\right) .
$$

In particular, $\sigma(x y)=\sigma(x) \sigma(y)$ for all $x, y \in \mathbb{Q}(i)$. We now apply $\sigma$ on Equation 5 .

$$
\begin{array}{r}
\left(1, \sigma(\zeta), \ldots, \sigma\left(\zeta^{2^{n-2}-1}\right)\right) M_{x}= \\
\left(\sigma(x), \sigma(x) \sigma(\zeta), \ldots, \sigma(x) \sigma\left(\zeta^{2^{n-2}-1}\right)\right),
\end{array}
$$

The matrix $M_{x}$ is unchanged since $\sigma$ is $\mathbb{Q}(i)$-linear. Thus $\sigma(x)$ is also an eigenvalue of $M_{x}$. By applying iteratively $\sigma$, we get that $\sigma^{j}(x), j=0, \ldots, m-1$ are eigenvalues of $M_{x}$. Note that we have exactly $m$ eigenvalues since $\sigma^{m}(x)=x$, by definition of $r$ (see Equation (6)).

We have thus proved

Proposition 1: Let $M_{x}$ be the matrix of multiplication by $x$. We have

$$
\operatorname{det}\left(M_{x}\right)=\prod_{j=0}^{m-1} \sigma^{j}(x) .
$$

To get full diversity, we finally notice that if $x \neq 0$, then $\sigma(x) \neq 0$. Indeed, since $\sigma(x)=\sum_{j=0}^{m-1} x_{j} \sigma\left(\zeta^{j}\right)$, we have by definition of basis that $\sigma(x)=0$ if and only if $x_{j}=0$ for all $j$.

The distributed space-time code thus satisfies the full diversity property.

We conclude this section by discussing some improvement issues. Notice first that a choice of

$$
G=\left(\begin{array}{ccccc}
0 & 0 & 0 & & \alpha \\
1 & 0 & 0 & & \\
0 & 1 & & & \\
\vdots & & \ddots & \ddots & \\
& & & 1 & 0
\end{array}\right),
$$

with $\alpha \neq i$ is also possible. In order for $G$ to be unitary, it is enough to choose $\alpha$ such that $|\alpha|^{2}=1$. However, proving the full diversity will be less systematic, since a particular value of $\alpha$ may be required for each dimension. Furthermore, if $\alpha \neq i$, then $\alpha=\alpha_{1} / \alpha_{2} \in \mathbb{Q}(i)$. Thus we can rewrite $\operatorname{det}\left(M_{y}\right)=\left(\frac{1}{\alpha_{2}}\right)^{m-1} \operatorname{det}\left(\tilde{M}_{y}\right)$, where $\tilde{M}_{y}$ is a matrix whose coefficients are in $\mathbb{Z}[i]$, obtained from $M_{y}$ by putting $\frac{1}{\alpha_{2}}$ in factor. Note that the denominator grows exponentially in $m-1$, since $\alpha$ appears in $m-1$ rows of $M_{y}$. Since the signal transmitted is carved from a QAM-constellation, all coefficients of $M_{y}$ are elements of $\mathbb{Z}[i]$, implying that $\operatorname{det}\left(\tilde{M}_{y}\right) \in \mathbb{Z}[i]$. Thus $\left|\left(\frac{1}{\alpha_{2}}\right)^{m-1} \operatorname{det}\left(\tilde{M}_{y}\right)\right|^{2}$ is lower bounded by $\left(\frac{1}{\left|\alpha_{2}\right|^{2}}\right)^{m-1}$. The bound on the pairwise error probability is then tighter by choosing $\alpha=i$. 


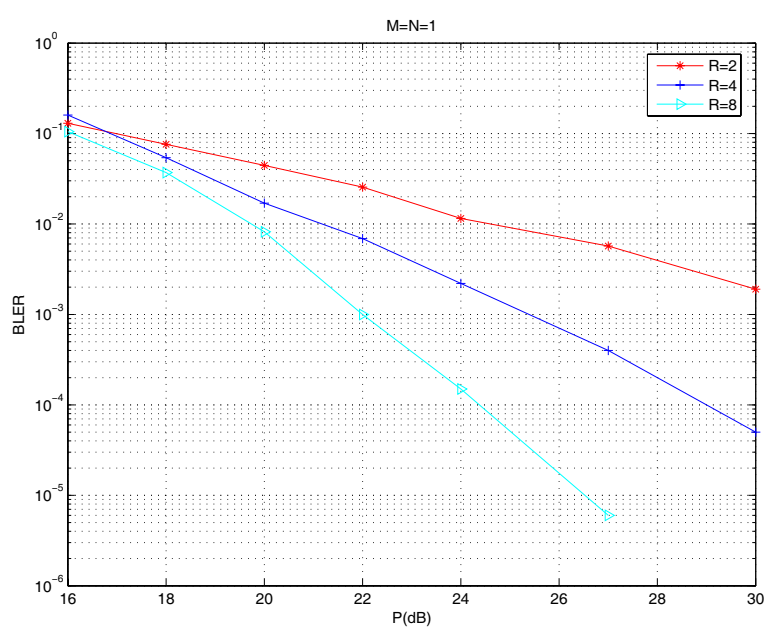

Fig. 2. Block Error Rate for $R=2,4,8$ relay nodes.

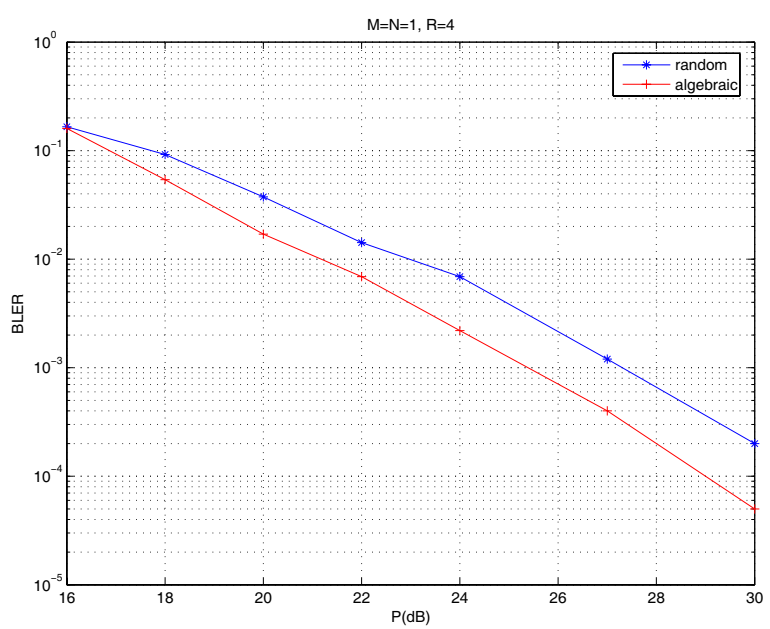

Fig. 3. For $T=R=4$ nodes, comparison between random and algebraic coding.

\section{Simulation RESUlts}

In this section, we present the simulation results. We are interested in comparing the performance of the space-time code described above to random codes used in [5]. The fading coefficients $f_{i}$ resp. $g_{i}$ between the transmitter and the relays, resp. the relays and the receiver, as well as the noises $\mathbf{v}$ and $\mathbf{w}$, are modeled as independent complex Gaussian variables with zero mean and unit variance. The fading coefficients are assumed constant for $T$ channel uses. The signal $s_{1}, \ldots, s_{T}$ are designed as independent 4-QAM signals. The decoding is done using Sphere decoding. The plots show the block error rate as a function of the power of the system. On Fig. 2, the block error rate for $T=R=2,4,8$ relay nodes is shown. The diversity of the system, as recalled in the introduction, is linear in $R$, which can be checked on this figure.

On Fig. 3, we compare the codes presented in this paper with random codes. By random codes, we mean that unitary random matrices are generated before transmission, and given to the nodes. It can be seen that both the codes achieve full diversity (the slopes are parallel). However, the algebraic code yields a much better coding gain.

Note also that the algebraic codes give better performance with much less encoding complexity, since the nodes essentially need to perform a shift, with a multiplication by $i$. Random coding asks for multiplication by full unitary matrices.

\section{CONCLUSION}

In this paper, we constructed an explicit algebraic family of distributed space-time that are proved to be fully diverse. The main required property was that the basis matrices $A_{i}$ be unitary. The performance of the code appears to be better than the one of the random codes considered in [5]. Though both random and algebraic codes achieve full diversity, algebraic codes give a better coding gain, with even less encoding complexity.

\section{REFERENCES}

[1] H. Blcskei, R. U. Nabar, . Oyman, and A. J. Paulraj, "Capacity scaling laws in MIMO relay networks", IEEE Trans. Wireless Communications, June 2006, to appear.

[2] Y. Chang and Y. Hua, "Application of space-time linear block codes to parallel wireless relays in mobile ad hoc networks", Thirty-Sixth Asilomar Conference on Signals, Systems and Computers, Nov 2003

[3] B. Hassibi and B. Hochwald, "High-rate codes that are linear in space and time", IEEE Transactions on Information Theory, vol 47, no 6, pp. 2335-2367, Sep 2002

[4] M. Janini, A. Hedayat, T.E. Hunter and A. Nosratinia, "Coded cooperation in wireless communications: Space-time transmission and iterative decoding", IEEE Transactions on Signal Processing, vol 52, no 2, pp. 362-371, Feb 2004

[5] Y. Jing and B. Hassibi, "Distributed space-time coding in wireless relay networks" to appear in the IEEE Transactions on Wireless Communications, 2006

[6] J.N. Laneman and G.W. Wornell, "Distributed space-time coded protocols for exploiting cooperative diversity in wireless networks", IEEE Transactions on Information Theory, vol 49, pp2415-2415, Oct 2003

[7] Y. Li and X.G. Xia, "A family of distributed space-time trellis codes with asynchronous cooperative diversity", Fourth International Symposium on Information Processing in Sensor Networks, pp. 340-347, Apr 2005

[8] R. Nabar, H. Bolcskei and F.W. Kneubuhler, "Fading relay channels: Performance limits and space-time signal design", IEEE Journal on Selected Areas in Communications, vol 22, no 6, pp. 1099-1199, Aug 2004

[9] A. Sendonaris, E. Erkip and B. Aazhang, "User cooperation diversityPart I: System Description", IEEE Transactions on Communications, vol 51, pp. 1927-1938, Nov 2003

[10] A. Sendonaris, E. Erkip and B. Aazhang, "User cooperation diversityPart I: Implemantaion aspects and performance analysis", IEEE Transactions on Communications, vol 51, pp. 1939-1948, Nov 2003

[11] H. Skjevling, D. Gesbert and A. Hjorungnes, "Precoded distributed space-time block codes in cooperative diversity-based downlink", submitted to the IEEE Transactions on Wireless Communications, Dec 2005

[12] Y. Tang and M.C. Valenti, "Coded transmit macro-diversity: Block space-time codes over distributed antennas", Vehicular Technology Conference (VTC), vol 2, pp. 1435-1438, May 2001 